\title{
EXPERIENCIAS DOCENTES EN LA IMPLEMENTACIÓN DE ESTRATEGIAS METODOLÓGICAS PARA LA COMPRENSIÓN LECTORA
}

Hentz Dona Contreras Tapahuasco

\begin{abstract}
RESUMEN
La investigación acción pedagógica constituye un eje muy fundamental para la práctica pedagógica, afín de guiar el quehacer docente a través de diseños de estrategias metodológicas. En el presente documento se realizara una revisión de los conceptos sobre las estrategias para la comprensión lectora, la práctica docente enfocada desde la investigación acción pedagógica y las mejoras de los procesos de aprendizaje y enseñanza.
\end{abstract}

\section{PALABRAS CLAVE}

Reconstrucción, deconstrucción, categorías, comprensión, estrategia metodológica.

\section{ABSTRACT}

The present research pedagogical action represents a very fundamental axis for the pedagogical practice, in order to manage the teaching task through the design of methodological strategies. This document will review the concepts of strategies for reading comprehension, teaching practice focused on research pedagogical action and improvement of learning and teaching processes.

\section{KEYWORDS}

Reconstruction, deconstruction, categories, comprehension, methodological strategy. $\square$ 1 presente artículo hace referencia a la recopilación de las prácticas docentes realizadas en el departamento de Ayacucho, responsable de dichas acciones fue la Universidad Nacional de San Cristóbal de Huamanga en convenio con el Ministerio de Educación. Mi persona estuvo a cargo de las Instituciones Educativas "José Abelardo Quiñones Gonzáles", "San Martín de Porres", ubicadas en el distrito de San Juan Bautista. Estas actividades se ejecutaron entre los años 2012 al 2015, en dichas instituciones se implementó el programa, orientado desde la investigación acción pedagógica con la finalidad de mejorar la práctica docente y las estrategias en la comprensión lectora en los estudiantes del nivel primario.

Está demostrado que el conocimiento, selección y adecuada aplicación de estrategias metodológicas por parte del docente, cumplen una función importante en la activación y desarrollo del aprendizaje en los estudiantes; sin embargo, cabe resaltar que en las últimas décadas los docentes no fueron capacitados, es a partir de estas deficiencias, que se implementa el Programa de Segunda Especialidad en Didáctica de la Educación Primaria, con la finalidad de mejorar la experiencia docente a través de la investigación acción pedagógica, y que se evidencian en las tesis elaboradas por los docentes a partir de la deconstrucción, reconstrucción y validación de la efectividad de la práctica alternativa o reconstruida, demostrándose así los cambios de la práctica pedagógica en los docentes. Para ello partiremos de las aportaciones teóricas existentes. 


\section{- Conceptos y teorías}

\section{Investigación acción pedagógica}

Se realiza sobre la práctica personal, es decir una investigación que se inicia y se reconstruye permanentemente en las actividades propias del maestro con la finalidad de elevar la calidad e incidencia de la formación de sus estudiantes. Al respecto, Elliot (2000), lo relaciona con los problemas prácticos cotidianos experimentados por los profesores, en vez de con los "problemas teóricos”. El propósito de la investigación-acción consiste en profundizar la comprensión del profesor (diagnóstico) de su problema. Por tanto, adopta una postura exploratoria frente a cualesquiera definiciones iniciales de su propia situación que el profesor pueda mantener.

La investigación acción tiene como soporte teórico el enfoque metodológico de la investigación cualitativa, apoyado en la investigación acción en el aula, en la cual se llevan a cabo tres fases: la deconstrucción, la reconstrucción y la evaluación de la efectividad de la práctica, que se repiten cíclicamente, cuya finalidad es la mejora de la práctica pedagógica.

La primera fase es la deconstrucción de la práctica que debe terminar en conocimiento profundo y una comprensión absoluta de la estructura de la práctica, respecto a los fundamentos teóricos, sus fortalezas y debilidades, siendo el punto de partida para la transformación del quehacer docente. Al respecto, Restrepo (2011) señala la implicación de la autocrítica alrededor de la propia práctica. Esta fase se inicia con la recolección de la información de la práctica pedagógica, haciendo un análisis crítico reflexivo del trabajo que se estaba realizando con los estudiantes, bajo los principios de retrospección e introspección.

La segunda fase es la reconstrucción según Restrepo, sostiene que el lunar principal es la sustentación teórica de los cambios propuestos para mejorar la práctica y sus resultados en el aprendizaje de los estudiantes. En esta fase es oportuna ensamblar de manera holística una propuesta que recoja las ideas para mejorar la práctica, apoyadas en las teorías pedagógicas vigentes.

Finalmente, la tercera fase tiene que ver con la validación de la efectividad de la práctica alternativa o reconstruida; es decir, con la constatación de su capacidad práctica, para lograr bien los propósitos de la educación.

\section{Comprensión lectora}

Leer es una actividad compleja para entender e interpretar textos escritos, con diferentes intenciones y objetivos, para lo que se necesita recurrir al conocimiento del mundo y a los conocimientos textuales y dominar las habilidades de decodificación y las estrategias para procesar activamente el sentido y los significados del texto. Al respecto, Solé (2008), sostiene que dichas estrategias son procedimiento de alto nivel, que implican metas, planificación de acciones para su consecución y una permanente evaluación para ajustar o cambiar dichas acciones.

La lectura favorece y enriquece la comprensión de la vida permitiendo el conocimiento del mundo, facilitando la comprensión.

La comprensión de un texto implica que el lector adopte un pensamiento estratégico para captar las ideas contenidas en los diversos tipos de textos, permitiéndole llegar al objetivo final de la lectura. Asimismo, la comprensión lectora es un proceso cognitivo que permite interactuar al lector con el texto. Al respecto, Cortes y García (2010), sostienen que la comprensión lectora es un proceso de elaboración de significados, la cual depende simultáneamente de tres factores: los datos proporcionados por el texto, los conocimientos previos y las actividades que realiza ellector.

Para garantizar la comprensión de un texto es necesario el uso de estrategias, esto conlleva a que el lector logre captar la idea central de un texto, pasaje o párrafo; es decir, de entender su contenido esencial. 
Estrategias metodológicas para la comprensión lectora

Solé (2008), son procedimientos de carácter elevado que implica la presencia de objetivos que cumplir, la planificación de las acciones que se desencadenan para lograrlo así como su evaluación y posible cambio. Al respecto, Solé plantea tres momentos para la lectura.

La primera que hace referencia al antes de la lectura, el cual crea las condiciones necesarias, en este caso, de carácter efectivo. O sea el encuentro anímico de los interlocutores, cada cual con lo suyo: uno que expone sus ideas (el texto), y el otro que aporta su conocimiento previo motivado por interés propio. La siguiente fase es el durante, donde se realiza una lectura de reconocimiento, en forma individual, para familiarizarse con el contenido general del texto. Seguidamente, pueden leer en pares o pequeños grupos y luego intercambiar opiniones. Después de la lectura, Solé también ha señalado que el trabajo es más reflexivo y crítico.

En este proceso los docentes deben dar a los alumnos las pautas de cómo articular su comprensión de lo que han leído y de poner a prueba su validez, que la aplique a una situación nueva o que argumenten defendiéndola contra una aseveración opuesta.

En esta etapa no se trata solo de aplicar preguntas sino de relacionar los nuevos aprendizajes de la lectura con los conocimientos reales del alumno.

\section{- Análisis}

El Programa de Segunda Especialidad en Educación Primaria se inició en agosto del 2012 a responsabilidad de la Universidad Nacional San Cristóbal de Huamanga en convenio con el Ministerio de Educación, se implementó la formación docente en servicio con el fin de desarrollar y articular con el marco de buen desempeño docente y de acuerdo a la política educativa nacional y regional, con el objetivo de fortalecer las competencias y los desempeños docentes a través de procesos formativos que permiten lograr el dominio pedagógico disciplinar de su campo de acción profesional; competencias didácticas e investigativas e innovadoras para un manejo efectivo de los procesos pedagógicos interculturales, capacidad para establecer vínculos socio afectivos positivos con sus estudiantes y el desarrollo personal como base del desarrollo profesional docente.

Desde esta perspectiva los docentes iniciaron su formación desde un enfoque crítico reflexivo e intercultural crítica, la primera que pone énfasis en el desarrollo de su autonomía profesional y capacidad para investigar, innovar y reflexionar críticamente sobre su práctica pedagógica para autorregular su saber pedagógico. Y el segundo, que orienta la afirmación del docente como mediador del dialogo intercultural y con capacidad para indagar y proponer alternativas educativas oportunas al contexto sociocultural y sociolingüístico del país.

El maestro inicia su formación considerando a la escuela como espacio privilegiado para aprender desde la experiencia y desde la propia práctica pedagógica articulando la investigación y la práctica pedagógica como herramientas que permitirán desarrollar la reflexión de su práctica y la construcción de saberes pedagógicos enmarcados desde la deconstrucción y reconstrucción de su quehacer docente, vinculados con el acompañamiento pedagógico.

Esta práctica pedagógica se orienta en la reflexión y sistematización, evidenciándose en una primera fase con la realización de los diarios de campos investigativos y el análisis de carácter crítico reflexivo, donde lograron estructurar las categorías que representan las debilidades en el proceso pedagógico. Posteriormente, se estableció una segunda fase para la identificación y análisis del problema, que les permitió elaborar una propuesta pedagógica alternativa teniendo en cuenta el quehacer docente, las teorías y enfoques pedagógicos. En esta fase los docentes iniciaron con la clasificación y sistematización de las categorías a través de un mapa conceptual de reconstrucción con el propósito de formular los cambios que efectuaran de su práctica, sustentadas en las teorías explícitas en interacción con las teorías implícitas de la deconstrucción de su práctica pedagógica. Esto permitió el diseño y la propuesta de acciones de cambio con la intención de mejorar las estrategias 
metodológicas a partir del enfoque comunicativo textual que en principio reconoce la lectura como una práctica social que debe tomar parte de la vida escolar del aula. Se busca que los estudiantes aprendan a leer con propósitos sociales y no simplemente para leer una tarea escolar.

La importancia metodológica como parte de la investigación consistió en la instrumentalización de los métodos, técnicas y los instrumentos en el área de comunicación enfocados específicamente en la comprensión de textos escritos, estos conceptos fueron recogidos a partir de la teoría de Isabel Solé.

Es a partir de esta propuesta pedagógica que el docente se introduce en el proceso de la investigación acción de su práctica pedagógica con el objetivo de establecer aspectos importantes que interactúan, tales como: la justificación teórica, metodología, técnicas y la aplicación pertinente de los enfoques teóricos como el constructivismo, el enfoque por competencias y su aplicación como parte del saber pedagógico.

En una primera fase, los docentes iniciaron con la deconstrucción de la práctica pedagógica para identificar las fortalezas y debilidades de su quehacer pedagógico, a través del recojo de información mediante diez diarios de campo investigativos, lo que permitió la sistematización y la descripción retrospectiva, introspectiva y detallada de todos los sucesos relacionados con su práctica concernientes a las debilidades recurrentes y fortalezas en función de sus diarios de campo. Este proceso se llevó a cabo mediante un análisis de carácter critico - reflexivo evidenciando en un mapa de deconstrucción. Sin embargo, los docentes no realizaron esta transformación de su práctica de forma aislada, sino asignándole un acompañante pedagógico a 14 participantes, quienes realizaron 112 horas de visita al docente en el contexto del aula y 80 horas en los círculos de interaprendizaje colaborativo, además de contar con asesorías virtuales y clases presenciales; con el objetivo de orientar y colaborar con el docente en todos los procesos de la investigación acción, identificando el problema, diagnóstico, diseño de la propuesta pedagógica innovadora, ejecución y sistematización, además de desarrollar la reflexión crítica en los docentes sobre los procesos de aprendizaje y enseñanza, ayudándole en la deconstrucción de su práctica que es indispensable para proceder a una transformación, promoviendo en el docente el ejercicio de la reflexión crítica permanente con la finalidad de desarrollar habilidades investigativas, en búsqueda de alternativas pertinentes que respondan a las necesidades de los estudiantes.

En una segunda fase, en la reconstrucción de la práctica pedagógica se inició con la identificación y análisis del problema, facilitando al docente elaborar una propuesta pedagógica alternativa teniendo en cuenta el quehacer pedagógico, teorías y enfoques psicopedagógicos. Esta fase consistió en hacer una radiografía de los aspectos más recurrentes en el ejercicio docente y la vinculación de los problemas planteados inicialmente en la deconstrucción de su práctica pedagógica; es a partir de esto, que se sistematiza en un mapa conceptual de reconstrucción de las categorías y subcategorías que serán implementadas en los procesos de aprendizaje y enseñanza, a través de un diseño de propuesta de acciones de cambio en la implementación de estrategias para la mejora de la comprensión lectora de textos escritos en los estudiantes, la estrategia se implementó a partir de tres etapas: ante, durante y después de la lectura, cada una de estas fases fueron ejecutadas por los maestros mediante un diseño de acciones alternativas, planificadas en una sesión de aprendizaje teniendo en cuenta el enfoque comunicativo textual y las teorías psicopedagógicas.

Asimismo, en esta fase se ejecutó la evaluación mediante indicadores objetivos para los docentes (se evidencian en la participación de los estudiantes y el profesor en la clase y los logros alcanzados) y subjetivos (que no se evidencian o muestran con claridad: evaluación de proceso continuo, percepciones, sentimientos) para los estudiantes, con la finalidad de verificar la efectividad de la propuesta pedagógica.

Finalmente, realizaron la evaluación de la ejecución de la propuesta pedagógica alternativa, es decir la sistematización y validación de la 
información de resultados de acuerdo a la identificación de las debilidades y fortalezas de la práctica docente.

Es a partir de la reconstrucción de la práctica que se evidencia gradualmente en el logro de aprendizajes de los estudiantes y en la mejora de la práctica pedagógica; de tal manera, que el docente deja de lado la improvisación y las prácticas tradicionales en el cual no utilizaba las estrategias metodológicas. La última fase de la investigación tiene que ver con la validación de la efectividad de la práctica alternativa o reconstruida, en esta fase el docente evalúa los resultados del progresivo avance y mejora de las prácticas pedagógicas, demostrándose en el recojo de información a través de los diferentes instrumentos de evaluación, como los diarios de campos (que servirán de lente interpretativo de la vida en el aula y en la escuela acercándose al acontecer cotidiano de la práctica del docente, permitiendo entrar profundamente en la propia experiencia y ver desde diferentes puntos su labor), listas de cotejo y otros. La siguiente cita es el análisis de la experiencia docente. Huamani, M. (2015) indica: "Mi práctica pedagógica actualmente se viene evidenciando en un cambio notable, de manera secuencial y progresiva con el uso adecuado de las diferentes estrategias innovadoras, métodos didácticos y la evaluación pertinente.....".

\section{CONCLUSIONES}

$\varnothing \quad$ Se puede afirmar que la Investigación Acción Pedagógica es un método de estudio que busca conseguir resultados fiables y útiles para mejorar la práctica pedagógica, fundando la investigación en la propia participación del investigador.

$\varnothing \quad$ Que los docentes investigadores a partir de la autocrítica de su propia práctica pedagógica lograron cambios significativos en los procesos de enseñanza y aprendizaje, evidenciándose en la mejora de su labor educativa.

\section{REFERENCIAS}

Cortes, M. y García, F. (2010). Estrategias de comprensión lectoray producción textual. Lima, Perú: Ed. San Marcos E.I.R.L.

Huamani, M. (2015). Aplicación de la técnica oral para mejorar la comprensión lectora en los estudiantes del 5to grado "B" de la Institución Educativa "Abelardo
Quiñones Gonzales" del distrito de San Juan Bautista.

Elliot, J. (2000). La investigación Acción en Educación. http://es.slideshare.net /bibliotecaiuacj/la-investigacin-accin-eneducación 